\title{
Gauge Covariant Theory of the Generating Operator. I
}

\author{
V. S. Gerdjikov^ and A. B. Yanovski \\ Joint Institute for Nuclear Research, Dubna, SU-101000 Moscow,_USSR
}

\begin{abstract}
A gauge covariant formulation of the generating operator $(\Lambda$ operator) theory for the Zakharov-Shabat system is proposed. The operator $\tilde{\Lambda}$, corresponding to the gauge equivalent system in the pole gauge is explicitly calculated. Thus the unified approach to the nonlinear Schrödinger-type equations based on $\Lambda$ is automatically reformulated with the help of $\tilde{\Lambda}$ for the Heisenberg ferromagnet-type equations. Consequently, it is established that the conserved densities for the Heisenberg-ferromagnet-type equations are polynomial in $S(x)$ and its $x$-derivatives. Special attention is paid to the interrelation between the hierarchies of symplectic structures corresponding to the above mentioned families of gauge-equivalent equations. It is shown that the geometrical properties of the conjugated operator $\Lambda^{*}$ are gaugeindependent.
\end{abstract}

\section{Introduction}

It is well known that the inverse scattering method (ISM) relates to a given linear problem $L(q, \lambda)$, where $q(x)$ denotes a set of coefficient functions and $\lambda$ the spectral parameter, a class of exactly solvable nonlinear evolution equations (NLEEs). A paradigm of such a linear problem is the so-called Zakharov-Shabat system $[1,2]$ :

$$
\begin{gathered}
L(q, \lambda) \psi=\left(i \frac{d}{d x}-q-\lambda \sigma_{3}\right) \psi=0 \\
q=q_{+} \sigma_{+}+q_{-} \sigma_{-}, \quad q_{ \pm}(x) \in \mathbb{C} \\
\sigma_{+}=\left(\begin{array}{ll}
0 & 1 \\
0 & 0
\end{array}\right), \quad \sigma_{-}=\left(\begin{array}{ll}
0 & 0 \\
1 & 0
\end{array}\right), \quad \sigma_{3}=\left(\begin{array}{rr}
1 & 0 \\
0 & -1
\end{array}\right) .
\end{gathered}
$$

A number of physically important NLEEs, such as the nonlinear Schrödinger equation (NLSE):

$$
i \sigma_{3} q_{t}+q_{x x}+2 q\langle q, q\rangle=0, \quad\langle q, q\rangle=\frac{1}{2} \operatorname{tr} q^{2},
$$

* Present address: Institute of Nuclear Research and Nuclear Energy, Sofia, Bulgaria 
the sine-Gordon and the modified Korteweg de Vries equations ( $m \mathrm{KdVE}$ ) have been solved by means of it. The function $q(x)$ in (1.1) can be considered as a coordinate function on the space of the potentials $\mathscr{M}=\{q(x)\}$ which possesses a natural symplectic structure. All the above mentioned equations are Hamiltonian with respect to this structure.

In the case of the Zakharov-Shabat system it was conjectured for the first time that the ISM may be interpreted as a generalized Fourier transform, see [2]. As generalized exponents, there naturally appear the "squared" solutions $\Psi^{ \pm f}(x, \lambda)$ of the system (1.1) [see Eqs. (2.7) below]. The analog of the differentiation operator is the integro-differential operator $\Lambda_{+}$, defined by the requirement that all the functions $\Psi^{ \pm f}(x, \lambda)$ are its eigenfunctions. The most important property of the system $\left\{\Psi^{ \pm f}(x, \lambda), \lambda \in \mathbb{R}\right\}$ is probably its completeness which has been demonstrated for the first time in [3] and more rigorously proved in [4]. Just because of it the mapping from the set of potentials $\mathscr{M}$ to the set of the scattering data of the system (1.1) is unique and invertible. Indeed, expanding the potential $q(x)$ and its variation over the system $\left\{\Psi^{ \pm f}(x, \lambda), \lambda \in \mathbb{R}\right\}$ one obtains as coefficients the minimal set of scattering data $R_{\varrho}$ and its variations, respectively $[5,6]$ [see formulae (2.9-10) below]. These expansions together with the inversion formulae (2.4) are fundamental and stress the importance of the generating operator $\Lambda_{+}$for the NLEEs related to the linear problem (1.1).

Moreover, with the help of the operator $\Lambda_{+}$one is able to generate not only the class of the solvable equations but also their conservations laws, the hierarchy of symplectic structures on $\mathscr{M}$ etc., see [5-11]. We would like to note, however, that for the effectiveness of the approach it is crucial to have an explicit expression of $\Lambda_{+}$through the potential $q(x)$.

The set of the equations which can be solved by means of the linear problem (1.1) will be referred to as the NLS-type equations. It consists of the equations having a Lax representation:

$$
[L, M]=0, \quad M=\frac{\partial}{\partial t}+V\left(q, q_{x}, \ldots, \lambda\right), \quad V \in \operatorname{sl}(2, \mathbb{C}),
$$

with a convenient choice for the matrix $V$ and $L$ given by (1.1). In the papers [12, 13] it has been realized for the first time that as soon as the Lax representation (1.3) is invariant under the gauge transformations one can consider the equations possessing gauge-invariant $L-M$ pairs as equivalent as well. This fact has been used in $[12,13]$ to reveal some important examples of gauge-equivalent NLEEs. The gauge transformation itself can be regarded as a nonsingular mapping between the phase spaces of these equations.

The most celebrated examples of the gauge equivalence we are speaking about include: i) the $n$-wave and the chiral field hierarchies $[13,14]$; ii) the multicomponent NLSE with vanishing boundary conditions and the equations of the LandauLifshitz type ( $X X X$-case), [14]; iii) the NLSE on symmetric spaces and the corresponding generalized Heisenberg ferromagnet equations [15]. The Heisenberg ferromagnet equation (HFE) is gauge-equivalent to the NLSE (1.2) and has the form:

$$
2 i S_{t}=\left[S, S_{x x}\right], \quad S^{2}=\mathbb{1}, \quad \lim _{x \rightarrow \pm \infty} S(x)=\sigma_{3}
$$


$[S(x, t)$ is an $\mathrm{sl}(2, \mathbb{C})$-valued function]. Equation (1.4) was solved with the help of the following system, known as the Zakharov-Shabat system in a pole-gauge:

$$
\begin{gathered}
\tilde{L} \tilde{\psi}=\left(i \frac{d}{d x}-\lambda S\right) \tilde{\psi}=0, \quad S=\psi_{0}^{-1} \sigma_{3} \psi_{0}, \\
\tilde{\psi}=\psi_{0}^{-1} \psi, \quad \psi_{0}=\psi(x, t, \lambda=0), \quad \lim _{x \rightarrow+\infty} \psi_{0}(x, t)=\mathbb{1},
\end{gathered}
$$

$\psi$ being a solution of the system (1.1).

It is natural to consider that one can reformulate the $\Lambda$-operator theory from one gauge into another. In the present paper we shall show on the example of the system (1.5) that it actually can be done. The system (1.5) has been chosen for the reason that the theory related to the linear problem (1.1) is well known but the corresponding theory for the system (1.5) has not been constructed ${ }^{1}$.

As far as we know there are few papers discussing the $\Lambda$-operator theory and the gauge transformation as well, see for example $[18,19]$. In $[18]$ the role of $L$ is played by a scalar differential operator of order $n$ in an arbitrary gauge. The corresponding expressions for $\Lambda$ contains both the coefficients $q$ and the analog of $\psi_{0}$. In [19] an expression for the operator $\tilde{\Lambda}$ generating the class of the chiral-type equations has been proposed but its interrelation with the corresponding $n$-wave $\Lambda$-operator has not been discussed.

The paper is organized as follows: In Sect. 2 we present the basic facts of the $\Lambda$-operator theory for the system (1.1). We give expressions for the inversion formulae, see (2.4), and a definition for $\Psi^{ \pm f}(x, \lambda)$, which are covariant under the adjoint group action (Ad-covariant). In Sect. 3 we apply the gauge transformation $L \rightarrow \tilde{L}=\psi_{0}^{-1} L \psi_{0}$ to the operator $\Lambda$ and explicitly calculate $\tilde{\Lambda}$ and the quantity $\delta \tilde{q}=\psi_{0}^{-1} \delta q \psi_{0}$ by means of $S$ and $\delta S$ only. This gives us an opportunity to reformulate automatically all the results concerning NLS-type equations to the analogous ones for the HF-type equations in Sect. 4. As a consequence we establish that the conservation law densities of the HF-type equations are polynomial in $S$ and its $x$-derivatives. We also show how the corresponding hierarchies of symplectic structures are interrelated. In the last section we discuss the geometrical properties of the conjugated operator $\Lambda^{*}$ and show that they are gaugeindependent.

Some of these results have been announced in [17].

\section{Ad-Covariant Formulation of the Basic Facts from the Theory of the NLS-type Equations}

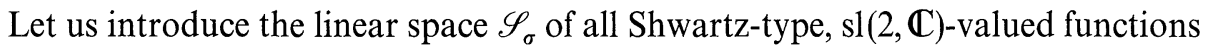
$q(x)$, satisfying the constraint $\left\langle q(x), \sigma_{3}\right\rangle=\frac{1}{2} \operatorname{tr} \sigma_{3} q=0$. Further we shall consider the linear problem (1.1) with $q \in \mathscr{S}_{\sigma}$, i.e., we choose the phase space $\mathscr{M}$ for the NLStype equations to be $\mathscr{S}_{\sigma}$. Below, for the sake of brevity and simplicity we assume that the linear problem (1.1) has no discrete spectrum. All the results however can be extended to the case when any finite number of eigenvalues with finite multiplicities are present, see, e.g., [4].

1 The situation with the $n$-wave hierarchy is similar but this case is more complex 
In what follows we shall need the Jost solutions and the transition matrix of the system $L \psi=0$ which are defined as follows:

$$
\begin{gathered}
\psi(x, \lambda)=\left\|\psi^{-}, \psi^{+}\right\|+\exp \left(-i \lambda \sigma_{3} x\right), \quad x \rightarrow+\infty, \\
\varphi(x, \lambda)=\left\|\varphi^{+}, \varphi^{-}\right\| \rightarrow \sigma_{3} \exp \left(-i \lambda \sigma_{3} x\right), \quad x \rightarrow-\infty, \\
T(\lambda)=\psi^{-1} \varphi=\left(\begin{array}{rr}
a^{+}(\lambda) & b^{-}(\lambda) \\
b^{+}(\lambda) & -a^{-}(\lambda)
\end{array}\right), \quad \operatorname{det} T=-1 .
\end{gathered}
$$

The existence and the analyticity properties of the Jost solutions are well known, see [2]. In (2.1) the superscript $+(-)$ everywhere except for $b^{+}, b^{-}$ indicates that the corresponding function is analytic in $\lambda$ for $\operatorname{Im} \lambda>0(\operatorname{Im} \lambda<0)$.

We recall some facts from the scattering theory of the system (1.1). First, one can choose as a minimal set of scattering data one of the following sets:

$$
\begin{aligned}
& R_{\varrho}=\left\{\varrho^{ \pm}(\lambda)=\frac{b^{ \pm}(\lambda)}{a^{ \pm}(\lambda)}, \lambda \in \mathbb{R}\right\}, \\
& R_{\tau}=\left\{\tau^{ \pm}(\lambda)=\frac{b^{\mp}(\lambda)}{a^{ \pm}(\lambda)}, \lambda \in \mathbb{R}\right\} .
\end{aligned}
$$

(It can be shown that they are equivalent.) The matrix $T(\lambda)$ can be reconstructed from $R_{\varrho}\left(R_{\tau}\right)$ by virtue of the dispersion relations, [2]:

$$
\begin{gathered}
\log a^{+}(\lambda)=\frac{i}{2 \pi} \int_{-\infty}^{+\infty} \frac{d \zeta}{\zeta-\lambda} \log \left(1+\varrho^{+} \varrho^{-}(\zeta)\right), \quad \operatorname{Im} \lambda>0, \\
\log a^{-}(\lambda)=-\frac{i}{2 \pi} \int_{-\infty}^{+\infty} \frac{d \zeta}{\zeta-\lambda} \log \left(1+\varrho^{+} \varrho^{-}(\zeta)\right), \quad \operatorname{Im} \lambda<0, \\
\log a^{+}(\lambda)\left(a^{-}(\lambda)\right)^{-1}=\text { p.v. } \frac{i}{\pi} \int_{-\infty}^{+\infty} \frac{d \zeta}{\zeta-\lambda} \log \left(1+\varrho^{+} \varrho^{-}(\zeta)\right), \quad \operatorname{Im} \lambda=0 .
\end{gathered}
$$

[Note that $\varrho^{+} \varrho^{-}(\zeta)=\tau^{+} \tau^{-}(\zeta), \zeta \in \mathbb{R}$.]

It was noticed, see [2] that the scattering data coefficients and their variations are expressed through so-called "squared" solutions $\Psi^{ \pm f}, \Phi^{ \pm f}$. Here we shall present these expressions in an Ad-covariant form. The words Ad-covariant mean covariant with respect to the adjoint group action $X \rightarrow g X g^{-1} \equiv \operatorname{Ad}(g) X$,

$$
\begin{aligned}
& \varrho^{ \pm}=-i\left(a^{ \pm}\right)^{-2} \int_{-\infty}^{+\infty}\left\langle\Phi^{ \pm},\left[\sigma_{3}, q\right]\right\rangle d x, \\
& \tau^{ \pm}=i\left(a^{ \pm}\right)^{-2} \int_{-\infty}^{+\infty}\left\langle\Psi^{ \pm},\left[\sigma_{3}, q\right]\right\rangle d x, \\
& \delta \varrho^{ \pm}= \pm 2 i\left(a^{ \pm}\right)^{-2} \int_{-\infty}^{+\infty}\left\langle\Phi^{ \pm}, \delta q\right\rangle d x, \\
& \delta \tau^{ \pm}= \pm 2 i\left(a^{ \pm}\right)^{-2} \int_{-\infty}^{+\infty}\left\langle\Psi^{ \pm}, \delta q\right\rangle d x, \\
& \Phi^{ \pm}=\varphi \sigma_{ \pm} \varphi^{-1}, \quad \Psi^{ \pm}=\psi \sigma_{\mp} \psi^{-1} .
\end{aligned}
$$


In the above expressions $\langle X, Y\rangle$ denotes the Killing form of the algebra $\mathrm{sl}(2, \mathbb{C})$, i.e., $\langle X, Y\rangle=\frac{1}{2} \operatorname{tr} X Y$. As the Killing form is invariant under the adjoint group action, Eqs. (2.4) are indeed written in a covariant way.

Let us remark in addition that the functions $\Phi^{ \pm}, \Psi^{ \pm}$satisfy the equation

$$
i \frac{d}{d x} \Phi^{ \pm}-\left[\lambda \sigma_{3}+q, \Phi^{ \pm}\right]=0
$$

which immediately follows from (2.5). This fact will appear in the explicit calculation of $\Lambda_{+}$.

From the formulae $(2.4 \mathrm{a}, \mathrm{b})$ it is easy to see that since the potential $q$ and its variation $\delta q$ are off-diagonal matrices one can write instead of $\Phi^{ \pm}$and $\Psi^{ \pm}$their off-diagonal parts $\Phi^{ \pm f}, \Psi^{ \pm f}$. Thus the decomposition $\Phi^{ \pm}=\Phi^{ \pm f}+\Phi^{ \pm d}$ (offdiagonal plus diagonal part) appears naturally here. In the case of the $\operatorname{sl}(2, \mathbb{C})$ algebra it can also be written in the following way:

$$
\Phi^{ \pm f}=\frac{1}{4}\left[\sigma_{3},\left[\sigma_{3}, \Phi^{ \pm}\right]\right], \quad \Phi^{ \pm d}=\left\langle\sigma_{3}, \Phi^{ \pm}\right\rangle \sigma_{3} .
$$

The formulae (2.4) show that the set of functions $\left\{\Psi^{ \pm f}(x, \lambda), \lambda \in \mathbb{R}\right\}$ and $\left\{\Phi^{ \pm f}(x, \lambda)\right.$, $\lambda \in \mathbb{R}\}$ are important in the scattering theory. $\operatorname{In}[3,6]$ it has been shown that they are complete sets, and hence one can expand over them every function $X(x) \in \mathscr{S}_{\sigma}$. The same is also true for the so-called symplectic basis $-\{P(x, \lambda), Q(x, \lambda), \lambda \in \mathbb{R}\}$, see [4]. $P$ and $Q$ are expressed through $\Psi^{ \pm f}, \Phi^{ \pm f}$ as follows:

$$
\begin{gathered}
P(x, \lambda)=\frac{1}{\pi}\left(\tau^{+} \Phi^{+f}-\tau^{-} \Phi^{-f}\right)=\frac{1}{\pi}\left(\varrho^{+} \Psi^{+f}-\varrho^{-} \Psi^{-f}\right), \\
Q(x, \lambda)=\frac{i}{2 b^{+} b^{-}}\left(\tau^{+} \Phi^{+f}-\varrho^{+} \Psi^{+f}\right)=\frac{i}{2 b^{+} b^{-}}\left(\tau^{-} \Phi^{-f}-\varrho^{-} \Psi^{-f}\right) .
\end{gathered}
$$

The importance of these sets is demonstrated by the following expansions, $[4,6]$ :

$$
\begin{aligned}
q(x) & =\frac{i}{\pi} \int_{-\infty}^{+\infty}\left(\varrho^{+} \Psi^{+f}-\varrho^{-} \Psi^{-f}\right) d \lambda \\
& =\frac{i}{\pi} \int_{-\infty}^{+\infty}\left(\tau^{+} \Phi^{+f}-\tau^{-} \Phi^{-f}\right) d \lambda \\
& =i \int_{-\infty}^{+\infty} P(x, \lambda) d \lambda, \\
\sigma_{3} \delta q= & -\frac{i}{\pi} \int_{-\infty}^{+\infty}\left(\delta \varrho^{+} \Psi^{+f}+\delta \varrho^{-} \Psi^{-f}\right) d \lambda \\
= & \frac{i}{\pi} \int_{-\infty}^{+\infty}\left(\delta \tau^{+} \Phi^{+f}+\delta \tau^{-} \Phi^{-f}\right) d \lambda \\
= & -\int_{-\infty}^{+\infty}(\delta \hat{q} P+\delta \hat{p} Q) d \lambda .
\end{aligned}
$$


The coefficients $\hat{p}, \hat{q}$ in the above formula are expressed through the scattering data of the system (1.1):

$$
\hat{q}=\frac{i}{2} \log \frac{b^{+}}{b^{-}}, \quad \hat{p}=-\frac{1}{\pi} \log \left(1+\varrho^{+} \varrho^{-}\right)
$$

and are known as the action-angle variables.

Now it is natural to introduce the operators $\Lambda_{ \pm}, \Lambda$ (if it is possible) through the requirements

$$
\left(\Lambda_{+}-\lambda\right) \Psi^{ \pm f}=\left(\Lambda_{-}-\lambda\right) \Phi^{ \pm f}=(\Lambda-\lambda) P=(\Lambda-\lambda) Q=0 .
$$

For the expansions over $\left\{\Psi^{ \pm f}\right\},\left\{\Phi^{ \pm f}\right\},\{P, Q\}$ these operators play the same role as the operator $\frac{d}{d x}$ for the Fourier expansion.

In order to show that one can actually construct such operators let us decompose [as in (2.7)] both sides of Eq. (2.6). Considering the diagonal part we have:

$$
\begin{gathered}
\Psi^{ \pm d}=-i \sigma_{3} \int_{+\infty}^{x} d y\left\langle\sigma_{3},\left[q, \Psi^{ \pm f}\right]\right\rangle, \\
\Phi^{ \pm d}=-i \sigma_{3} \int_{-\infty}^{x} d y\left\langle\sigma_{3},\left[q, \Phi^{ \pm f}\right]\right\rangle .
\end{gathered}
$$

Inserting (2.13) into the off-diagonal part of (2.6) yields:

$$
\Lambda_{ \pm}=\frac{i}{4}\left[\sigma_{3}, \frac{d}{d x} \cdot\right]-i q(x) \int_{ \pm \infty}^{x}\left\langle\sigma_{3},[q, \cdot]\right\rangle d y .
$$

Finally, it can be shown that $\Lambda$ in (2.12) equals $\frac{1}{2}\left(\Lambda_{+}+\Lambda_{-}\right)$.

We shall display now without proof the basic facts for NLS-type equations using notations convenient for our purpose.

I) The interpretation of the ISM as a generalized Fourier transform.

This point is clear enough because from the expansions (2.9) and the inversion formulae (2.4) it follows that the potential $q(x)$ is uniquely recovered from the set $R_{\ell}\left(\right.$ or $R_{\tau}$ ) and vice versa.

II) The class of NLS-type equations is given by:

$$
\frac{1}{2} F\left(\Lambda_{+}\right)\left[\sigma_{3}, q_{t}\right]+G\left(\Lambda_{+}\right) q=0,
$$

where the functions $F(\lambda)$ and $G(\lambda)$ are rational ${ }^{2}$ in $\lambda$. The NLEEs (2.15) are equivalent to the following linear equations for the scattering data $R_{\varrho}$ :

$$
F(\lambda) \varrho_{t}^{ \pm} \mp G(\lambda) \varrho^{ \pm}=0 .
$$

Remark 1. The same equations could be obtained if instead of $\Lambda_{+}$we inserted in

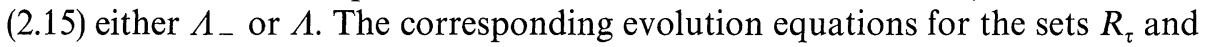

2 In order to ensure the existence of the inverse operators one must restrict the space $\mathscr{S}_{\sigma}$. For example, for the existence of the inverse operators $\Lambda_{ \pm}^{-1}$ it is sufficient to restrict ourselves to the submanifold $\mathscr{M}^{0} \subset \mathscr{S}_{\sigma}$ - see the explanation after (3.2) below 
$\{\hat{p}(\lambda), \hat{q}(\lambda)\}$ are

$$
\begin{gathered}
F(\lambda) \tau_{t}^{ \pm} \pm G(\lambda) \tau^{ \pm}=0, \\
\hat{p}_{t}=0, \quad \hat{q}_{t}=i G(\lambda) F(\lambda)^{-1} .
\end{gathered}
$$

In particular, if $F(\lambda)=1, G(\lambda)=4 i \lambda^{2}$ we get the NLSE (1.2). If one chooses some real form of the algebra $\operatorname{sl}(2, \mathbb{C})$, i.e., if one assumes that $q_{+}=q_{-}^{*}\left(q_{+}=-q_{-}^{*}\right)$ the NLSE with attraction (repulsion) could be obtained, see [1]. Other choices for the functions $F(\lambda)$ and $G(\lambda)$ lead to some well known equations. For example,

$$
F=(8 i)^{-1}, \quad G=\lambda^{3}
$$

leads to the complex mKdV equation:

$$
q_{t}+q_{x x x}+6 q_{x}\langle q, q\rangle=0
$$

(here one can obtain as above two different versions of the mKdVE by choosing some real form of the algebra). Similarly, the choice

$$
F=1, \quad G=-i \lambda^{-1},
$$

with the additional constraints

$$
q_{+}=\varepsilon q_{-}, \quad \varepsilon= \pm 1, \quad q_{+}=\frac{1}{2} v_{x},
$$

where $v(x, t)$ is a Shwartz-type function in $x$, leads to the sine-Gordon equation

$$
v_{t x}=\sin v
$$

for $\varepsilon=1$ and to the sinh-Gordon equation

$$
v_{x t}=\sinh v
$$

for $\varepsilon=-1$.

Remark 2. The conditions (2.20) imply that we restrict ourselves to the submanifolds $V_{ \pm}=\left\{\left(\sigma_{+} \pm \sigma_{-}\right) f_{x}(x)\right\}, V_{ \pm} \subset \mathscr{S}_{\sigma}$. These submanifolds are not invariant under the action of $\Lambda_{ \pm}$(or $\left.\Lambda\right)$. In fact, $\Lambda_{ \pm}\left(V_{ \pm}\right) \subset V_{\mp}$. Since the operator ad $\sigma_{3}=\left[\sigma_{3}, \cdot\right]$ has the same property, the evolution equations defined by:

$$
\frac{1}{2}\left[\sigma_{3}, q_{t}\right]+G\left(\Lambda_{+}\right) q=0
$$

will be self-consistent when restricted to $V_{+}$(or $V_{-}$) only if the function $G(\lambda)$ is odd. In other words, if we assume that the linear problem $L(q, \lambda) \psi=0$ allows the involution

$$
L(q, \lambda)=\tau_{0} L(q,-\lambda) \tau_{0}^{-1}, \quad \tau_{0}=\left(\begin{array}{ll}
0 & 1 \\
\varepsilon & 0
\end{array}\right),
$$

then from (2.1) it follows that $\varrho^{ \pm}(-\lambda)=-\varepsilon \varrho^{ \pm}(\lambda)$. Applying this to Eqs. (2.16) we get that $G$ is an odd function. In the same way from the dispersion relations (2.3) it follows that $\log a^{+}(\lambda)$ is also odd. form.

Note that the subspaces $V_{+}$and $V_{-}$are orthogonal with respect to the Killing 
III) Compact expressions for the conserved quantities are given by:

$$
\begin{gathered}
\log a^{+}(\lambda)=\sum_{m=1}^{\infty} c_{m} \lambda^{-m}, \\
c_{m}=-\frac{1}{m} \int_{-\infty}^{+\infty} d x \int_{+\infty}^{x}\left\langle\left[\sigma_{3}, q\right], \Lambda_{+}^{m} q\right\rangle d y .
\end{gathered}
$$

If the restrictions (2.20) hold, it is easy to see that from $q \in V_{+}$it follows that $\Lambda_{+}^{2 k} q \in V_{+}$. Since $V_{+}$and $V_{-}$are orthogonal $C_{2 k}=0, k=1, \ldots$. This is another way to prove that in this case $\log a^{+}(\lambda)$ is an odd function.

IV) The NLS-type equations are Hamiltonian ones with respect to an infinite number (hierarchy) of symplectic structures defined through the following 2-forms, $[6,8-11]$ :

$$
\begin{aligned}
\omega_{F}(X, Y) & =i \int_{-\infty}^{+\infty}\left\langle X, F(\Lambda)\left[\sigma_{3}, Y\right]\right\rangle d x \\
& =\int_{-\infty}^{+\infty} d \lambda F(\lambda) d \hat{p}(\lambda) \wedge d \hat{q}(\lambda)(X, Y) ; \quad X, Y \in \mathscr{S}_{\sigma} .
\end{aligned}
$$

(Here the symbol $\wedge$ means the exterior product.) The Hamiltonians of Eqs. (2.15) are linear combinations of the conserved quantities $c_{m}$. By virtue of the dispersion relations (2.3) one finds

$$
\begin{gathered}
H_{G}=2 \sum_{m=1}^{N} c_{m} G_{m}=i \int_{-\infty}^{+\infty} d \lambda G(\lambda) \hat{p}(\lambda), \\
G(\lambda)=\sum_{m=1}^{N} G_{m} \lambda^{m-1} .
\end{gathered}
$$

From Eqs. (2.26-27) it is clear that all Eqs. (2.15) are completely integrable Hamiltonian systems and the quantities $\hat{q}(\lambda), \hat{p}(\lambda)$ are indeed the corresponding action-angle variables.

\section{The Generating Operator and the Gauge Transformations}

Let us pass to the system (1.5) and see how such objects as $\Psi^{ \pm f}, \Phi^{ \pm f}, q, \delta q, \Lambda_{+}$etc. change under the gauge transformation: $L \rightarrow \tilde{L}=\psi_{0}^{-1} L \psi_{0}$. Here and below all the quantities related to (1.5) will be supplied with a tilde.

The Jost solutions $\tilde{\varphi}, \tilde{\psi}$, corresponding to the linear problem $\tilde{L} \tilde{\psi}=0$ are defined in a similar way as for the system (1.1) and are related to the functions $\varphi, \psi$, and $T$ as follows:

$$
\tilde{\psi}=\psi_{0}^{-1} \psi, \quad \tilde{\varphi}=\psi_{0}^{-1} \varphi T(0)^{-1} \sigma_{3}, \quad \tilde{T}(\lambda)=T(\lambda) T(0)^{-1} \sigma_{3} .
$$
For the HF equation there exist natural boundary conditions: $\lim _{x \rightarrow \pm \infty} S(x)=\sigma_{3}$.
It can be seen that they are not violated if

$$
T(0)=\left(\begin{array}{cc}
a^{+}(0) & 0 \\
0 & -a^{-}(0)
\end{array}\right), \text { i.e., } \quad \varrho^{ \pm}(0)=\tau^{ \pm}(0)=0 .
$$


The sets $R_{\varrho}$ and $R_{\tau}$ restricted by (3.2) will be referred to as $R_{\varrho}^{0}$ and $R_{\tau}^{0}$. In what follows we shall consider only a subclass of potentials $q(x)$ and a subclass of their variations $\delta q$ such that (3.2) together with $\delta \varrho^{ \pm}(0)=\delta \tau^{ \pm}(0)=0$ are fulfilled. The submanifold of these potentials we denote through $\mathscr{M}^{0}$. In its turn the phase space for the linear problem (1.5) is denoted by $\tilde{\mathscr{M}}$,

$$
\tilde{\mathscr{M}}=\left\{S(x): S^{2}=\mathbb{1}, \lim _{x \rightarrow \pm \infty} S(x)=\sigma_{3}, S(x) \in \operatorname{sl}(2, \mathbb{C})\right\}
$$

Remark 3. From (3.1) and (3.2) it follows that $\varrho^{ \pm}=\tilde{\varrho}^{ \pm}$, which ensures that the scattering problem for (1.1) and (1.5) are equivalent since they have the same minimal sets of scattering data $R_{\varrho}^{0}=\widetilde{R}_{\underline{\varrho}}$.

Let us define the matrices $\tilde{\Phi}^{ \pm}$and $\tilde{\Psi}^{ \pm}$as: $\tilde{\Phi}=\tilde{\varphi} \sigma_{ \pm} \tilde{\varphi}^{-1}, \tilde{\Psi}^{ \pm}=\tilde{\psi} \sigma_{\mp} \tilde{\psi}^{-1}$. From (3.1) we get:

$$
\tilde{\Phi}^{ \pm}=\psi_{0}^{-1} \Phi^{ \pm} \psi_{0}\left[a^{\mp}(0)\right]^{2}, \quad \widetilde{\Psi}^{ \pm}=\psi_{0}^{-1} \Psi^{ \pm} \psi_{0}
$$

Now our idea is to obtain the main results of the $A$-operator theory for the system (1.5) simply applying the transformation $X \rightarrow \psi_{0}^{-1} X \psi_{0}$ to the results (I-IV) for the linear problem (1.1). Following this thread we define the transformed system of "squared" solutions and the corresponding symplectic basis as follows:

$$
\begin{gathered}
\tilde{\Phi}^{ \pm f}=\tilde{\Phi}^{ \pm}-S\left\langle\tilde{\Phi}^{ \pm}, S\right\rangle, \quad \tilde{\Psi}^{ \pm f}=\tilde{\Psi}^{ \pm}-S\left\langle\tilde{\Psi}^{ \pm}, S\right\rangle, \\
\tilde{P}=\frac{1}{\pi}\left(\tilde{\tau}^{+} \tilde{\Phi}^{+f}-\tilde{\tau}^{-} \tilde{\Phi}^{-f}\right)=\frac{1}{\pi}\left(\tilde{\varrho}^{+} \tilde{\Psi}^{+f}-\tilde{\varrho}^{-} \tilde{\Psi}^{-f}\right), \\
\tilde{Q}=\frac{i}{2 \tilde{b}^{+} \tilde{b}^{-}}\left(\tilde{\tau}^{+} \tilde{\Phi}^{+f}-\tilde{\varrho}^{+} \bar{\Psi}^{+f}\right)=\frac{i}{2 \tilde{b}^{+} \tilde{b}^{-}}\left(\tilde{\tau}^{-} \tilde{\Phi}^{-f}-\tilde{\varrho}^{-} \tilde{\Psi}^{-f}\right) .
\end{gathered}
$$

Simultaneously we have defined the new decomposition

$$
\tilde{\Phi}^{f}=\frac{1}{4}\left[S,\left[S, \tilde{\Phi}^{ \pm}\right]\right], \quad \tilde{\Phi}^{ \pm d}=S\left\langle\tilde{\Phi}^{ \pm}, S\right\rangle .
$$

The prerequisite property of the operators $\Lambda_{ \pm}$is that $\Psi^{ \pm f}$ and $\Phi^{ \pm f}$ are their eigenfunctions. If we want the same property to hold for the operators $\tilde{\Lambda}_{ \pm}$and the functions $\widetilde{\Psi}^{ \pm f}, \widetilde{\Phi}^{ \pm f}$, then we must have: $\widetilde{\Lambda}_{ \pm}\left(\psi_{0}^{-1} X \psi_{0}\right)=\psi_{0}^{-1}\left(\Lambda_{ \pm} X\right) \psi_{0}, X \in \mathscr{S}_{\sigma}$. The most simple way of calculating $\tilde{\Lambda}_{ \pm}$is to make use of this relation. But in order to stress the similarity between the two different gauges we shall follow the same way as before. We look upon the gauge transformation as to an $x$-dependent change of the basis of the algebra $\operatorname{sl}(2, \mathbb{C})$. That is, we introduce the moving frame $\tilde{\sigma}_{3}, \tilde{\sigma}_{ \pm}$and the corresponding covariant derivative by:

$$
\begin{gathered}
\tilde{\sigma}_{3}=S=\psi_{0}^{-1} \sigma_{3} \psi_{0}, \quad \tilde{\sigma}_{ \pm}=\psi_{0}^{-1} \sigma_{ \pm} \psi_{0} \\
\nabla_{x} \tilde{X}=\frac{d}{d x} \tilde{X}+\left[\psi_{0}^{-1} \psi_{0 x}, \tilde{X}\right]
\end{gathered}
$$

Evidently, one has $\nabla_{x} \tilde{\sigma}_{\alpha}=0$, and as a consequence

$$
\nabla_{x}^{-1}\left(\sum_{\alpha} Q_{\alpha}(x) \tilde{\sigma}_{\alpha}\right)=\sum_{\alpha} \tilde{\sigma}_{\alpha} \int^{x} d y Q_{\alpha}(y) .
$$


[Here $Q_{\alpha}(x)$ are $\mathbb{C}$-valued functions and $\alpha$ runs over,,+- 3 .]

We must decompose now the equation:

$$
i \frac{d}{d x} \tilde{\Phi}^{ \pm}-\lambda\left[S, \tilde{\Phi}^{ \pm}\right]=0
$$

which is the analog of Eq. (2.6) after the gauge transformation. In this case the basis $\tilde{\sigma}_{\alpha}$ is $x$-dependent and $\frac{d}{d x}$ does not respect the decomposition $\tilde{\Phi}=\tilde{\Phi}^{f}+\tilde{\Phi}^{d}$. We must use then the covariant derivative $\nabla_{x}$, which can be expressed through $S$ because of the following relation:

$$
i \psi_{0}^{-1} \psi_{0 x}=\frac{i}{4}\left[S, S_{x}\right]=\tilde{q} \equiv \psi_{0}^{-1} q \psi_{0}
$$

Decomposing (3.8) we get:

$$
\begin{gathered}
i \nabla_{x} \tilde{\Phi}^{d}=\left[\tilde{q}, \tilde{\Phi}^{f}\right], \\
i \nabla_{x} \tilde{\Phi}^{f}=\left[\tilde{q}, \tilde{\Phi}^{d}\right]+\lambda\left[S, \tilde{\Phi}^{f}\right] .
\end{gathered}
$$

Next, keeping in mind (3.7), one can express $\tilde{\Phi}^{d}$ from the first of these equations:

$$
\tilde{\Phi}^{ \pm d}=S \int_{-\infty}^{x}\left\langle S_{y}, \tilde{\Phi}^{ \pm f}\right\rangle d y, \quad \tilde{\Psi}^{ \pm d}=S \int_{+\infty}^{x}\left\langle S_{y}, \tilde{\Psi}^{ \pm f}\right\rangle d y .
$$

Inserting after that (3.11) into the second line of (3.10), we obtain the necessary formulae for the operators $\tilde{\Lambda}_{ \pm}$:

$$
\tilde{\Lambda}_{ \pm} \tilde{X}=\frac{i}{4}\left[S, \frac{d}{d x} \tilde{X}\right]+\frac{i}{4}\left[S, S_{x}\right] \int_{ \pm \infty}^{x}\left\langle S_{y}, \tilde{X}(y)\right\rangle d y .
$$

Finally, the operator $\tilde{\Lambda}$ whose eigenfunctions are the elements of the symplectic basis (3.5) is again given by $\frac{1}{2}\left(\tilde{\Lambda}_{+}+\tilde{\Lambda}_{-}\right)$. Let us note that in (3.12) $\tilde{X}$ stands for an arbitrary vector from the space $\mathscr{S}_{S}$, consisting of all sl $(2, \mathbb{C})$-valued Shwartz-type functions $\tilde{X}(x)$ satisfying the condition $\langle S(x), \tilde{X}(x)\rangle=0$.

Remark 4. It is clear that the matrix functions $\tilde{\Psi}^{ \pm f}, \tilde{\Phi}^{ \pm f}$ have the same analyticity properties as $\Psi^{ \pm f}, \Phi^{ \pm f}$. Moreover, the completeness of the sets $\left\{\tilde{\Phi}^{ \pm f}\right\},\left\{\tilde{\Psi}^{ \pm f}\right\}$, and $\{\tilde{P}, \tilde{Q}\}$ in $\mathscr{S}_{S}$ is an immediate consequence of the completeness of the sets $\left\{\Phi^{ \pm f}\right\}$, $\left\{\Psi^{ \pm f}\right\}$, and $\{P, Q\}$ in $\mathscr{S}_{\sigma}$.

Below we shall need the following symmetric and skew symmetric ${ }^{3}$ nondegenerate bilinear forms on the spaces $\mathscr{S}_{\sigma}$ and $\mathscr{S}_{S}$ :

$$
\begin{gathered}
g(X, Y)=\int_{-\infty}^{+\infty}\langle X, Y\rangle d x, \quad X, Y \in \mathscr{S}_{\sigma}, \\
\omega_{0}(X, Y)=i \int_{-\infty}^{+\infty}\left\langle X,\left[\sigma_{3}, Y\right]\right\rangle d x, \quad X, Y \in \mathscr{S}_{\sigma},
\end{gathered}
$$

3 The 2-forms $\omega_{0}$ and $\tilde{\omega}_{0}$ are in fact symplectic ones and have been used in [4-6,8] and in [12] to describe the Hamiltonian structures of the NLS and HF equations, respectively 


$$
\begin{gathered}
\tilde{g}(\tilde{X}, \tilde{Y})=\int_{-\infty}^{+\infty}\langle\tilde{X}, \tilde{Y}\rangle d x, \quad \tilde{X}, \tilde{Y} \in \mathscr{S}_{S}, \\
\tilde{\omega}_{0}(\tilde{X}, \tilde{Y})=i \int_{-\infty}^{+\infty}\langle\tilde{X},[S, \tilde{Y}]\rangle d x, \quad \tilde{X}, \tilde{Y} \in \mathscr{S}_{S} .
\end{gathered}
$$

The following two lemmas are directly verified.

Lemma 1. The operators conjugated to $\Lambda_{ \pm}$and $\tilde{\Lambda}_{ \pm}$with respect to the forms $g$ and $\tilde{g}$ are given by the expressions:

$$
\begin{aligned}
& \Lambda_{ \pm}^{*} X=\frac{i}{4}\left[\sigma_{3}, \frac{d}{d x} X\right]+i\left[\sigma_{3}, q\right] \int_{\mp \infty}^{x}\langle q, X\rangle d y, \quad X \in \mathscr{S}_{\sigma}, \\
& \tilde{\Lambda}_{ \pm}^{*} \tilde{X}=\frac{i}{4}\left[S, \frac{d}{d x}\right]-\frac{i}{4} S_{x} \int_{\mp \infty}^{x}\left\langle\left[S, S_{y}\right], \tilde{X}\right\rangle d y, \quad \tilde{X} \in \mathscr{S}_{S} .
\end{aligned}
$$

Lemma 2. The operators $\Lambda_{+}^{*}$ and $\Lambda_{-}^{*}$ are conjugated with respect to the form $\omega_{0}$, so are the operators $\tilde{\Lambda}_{+}^{*}$ and $\tilde{\Lambda}_{-}^{*}$ with respect to the form $\tilde{\omega}_{0}$ :

$$
\begin{array}{ll}
\omega_{0}\left(X, \Lambda_{ \pm}^{*} Y\right)=\omega_{0}\left(\Lambda_{\mp}^{*} X, Y\right), & X, Y \in \mathscr{S}_{\sigma}, \\
\tilde{\omega}_{0}\left(\tilde{X}, \tilde{\Lambda}_{ \pm}^{*} \tilde{Y}\right)=\tilde{\omega}_{0}\left(\tilde{\Lambda}_{\mp}^{*} \tilde{X}, \tilde{Y}\right), & \tilde{X}, \tilde{Y} \in \mathscr{S}_{S} .
\end{array}
$$

Remark 5 . It is easy to see that $\tilde{\Lambda}_{ \pm}^{*}$ are gauge equivalent to $\Lambda_{ \pm}^{*}$.

Next, keeping in mind the nondegeneracy of the scalar products $g$ and $\tilde{g}$ one can prove one more lemma.

Lemma 3. The following relations hold:

$$
\begin{gathered}
\operatorname{ad} \sigma_{3} \Lambda_{ \pm}^{*}=\Lambda_{\mp} \operatorname{ad} \sigma_{3}, \\
\operatorname{ad} S \tilde{\Lambda}_{ \pm}^{*}=\tilde{\Lambda}_{\mp} \operatorname{ad} S, \\
\operatorname{ad} \sigma_{3} \Lambda^{*}=\Lambda \operatorname{ad} \sigma_{3}, \\
\operatorname{ad} S \tilde{\Lambda}^{*}=\tilde{\Lambda} \operatorname{ad} S .
\end{gathered}
$$

These relations will appear in Sect. 5 as one of the characteristic properties of the operator $\Lambda^{*}$. They are useful in some calculations as well.

Now, our aim is to obtain expansions like (2.4), and for this it is important to find an expression of the quantity $\delta \tilde{q}=\psi_{0}^{-1} \delta q \psi_{0}$ through the variation of the potential $S(x)$ in (1.5). The next lemma shows how it can be done:

\section{Lemma 4.}

$$
\begin{gathered}
\delta \tilde{q}=\operatorname{Ad}\left(\psi_{0}^{-1}\right) \delta q=\tilde{\Lambda}_{-}^{*}(\delta S), \\
\delta \tilde{q}=\tilde{\Lambda}_{+}^{*}(\delta S)-i S_{x} \delta\left(\log a^{+}(0)\right) .
\end{gathered}
$$

Let us first make some comments about the notations we use. The variation $\delta q$ belongs to $\mathscr{S}_{\sigma}$ and is considered as a tangent vector at the point $q \in \mathscr{S}_{\sigma}$. Quantities like $S, a^{+}(\lambda), \psi_{0}$ are mappings of the type $F: \mathscr{S}_{\sigma} \rightarrow V$, where $V$ is some linear space. Then for the sake of brevity we sometimes denote through $\delta F$ the Gateau derivative at the point $q$ along the vector $\delta q$, i.e., $\delta F(q)=F^{\prime}(q)(\delta q)$. In the special case $V=\mathbb{C}, \mathbb{R}$ we write $d F$ instead of $F^{\prime}$. 
Proof of Lemma 4. From the definition of $S(x)$, see (1.5), it follows that $\delta S=\left[S, \psi_{0}^{-1} \delta \psi_{0}\right]=\operatorname{Ad}\left(\psi_{0}^{-1}\right)\left[\sigma_{3}, \delta \psi_{0} \psi_{0}^{-1}\right]$, and hence

$$
\operatorname{Ad}\left(\psi_{0}^{-1}\right)\left(\delta \psi_{0} \psi_{0}^{-1}\right)^{f}=\frac{1}{4}[S, \delta S] .
$$

Let us consider now the matrix function $A(x, \lambda)=\delta \psi \psi^{-1}(x, \lambda), \psi$ being the Jost solution introduced in $(2.1)\left(A(x, 0)=\delta \psi_{0} \psi_{0}^{-1}\right)$. From (1.1) we obtain that $A(x, \lambda)$ satisfies the equation:

$$
i \frac{d}{d x} A-\left[\lambda \sigma_{3}+q, A\right]=\delta q .
$$

Separating the diagonal and the off-diagonal parts of (3.28) and taking some care of the integration constants one is able to find that

$$
\delta q=\left[\sigma_{3},\left(\Lambda_{ \pm}-\lambda\right) A^{f}(x, \lambda)\right]+\left[\sigma_{3}, q\right] \lim _{x \rightarrow \pm \infty}\left\langle\sigma_{3}, A(x, \lambda)\right\rangle .
$$

We shall need this relation only for $\lambda=0$. In this case the limits are easily calculated:

$$
\lim _{x \rightarrow+\infty}\left\langle\sigma_{3}, A(x, 0)\right\rangle=0, \quad \lim _{x \rightarrow-\infty}\left\langle\sigma_{3}, A(x, 0)\right\rangle=-\delta \log a^{+}(0) .
$$

Finally, we apply $\operatorname{Ad}\left(\psi_{0}^{-1}\right)$ to the both sides of (3.29), $(\lambda=0)$ and as from the definition of the operators $\tilde{\Lambda}_{ \pm}$it follows that

$$
\operatorname{Ad}\left(\psi_{0}^{-1}\right) \Lambda_{ \pm}=\tilde{\Lambda}_{ \pm} \operatorname{Ad}\left(\psi_{0}^{-1}\right),
$$

we have

$$
\begin{aligned}
\delta \tilde{q} & =\operatorname{Ad}\left(\psi_{0}^{-1}\right) \delta q=\left[S, \tilde{\Lambda}_{+} \operatorname{Ad}\left(\psi_{0}^{-1}\right) A^{f}\right. \\
& =\left[S, \tilde{\Lambda}_{-} \operatorname{Ad}\left(\psi_{0}^{-1}\right) A^{f}\right]-[S, \tilde{q}] \delta \log a^{+}(0) .
\end{aligned}
$$

Now, inserting the right-hand side of (3.27) in these formulae and using Lemma 3 we arrive at the expressions (3.25-26).

It is useful to reformulate the above lemma in a somewhat different way:

The Gateau derivative $q^{\prime}(S)$ of the mapping $q:(q=q(S))$ is given by the formulae:

$$
\begin{aligned}
q^{\prime}(S) & =\operatorname{Ad}\left(\psi_{0}\right) \tilde{\Lambda}_{-}^{*}=\operatorname{Ad}\left(\psi_{0}\right) \tilde{\Lambda}_{+}^{*}-i \operatorname{Ad}\left(\psi_{0}\right) S_{x} d \log a^{+}(0) \\
& =\operatorname{Ad}\left(\psi_{0}\right) \tilde{\Lambda}^{*}-\frac{i}{2} \operatorname{Ad}\left(\psi_{0}\right) S_{x} d \log a^{+}(0)
\end{aligned}
$$

\section{The A-Operator Theory for the HF Type Equations}

The results of the previous section help us to establish two theorems which are analogs to the relations (2.4) and (2.9-10). 
Theorem 1. The following inversion formulae hold:

$$
\begin{gathered}
\tilde{\varrho}^{ \pm}=\left(\tilde{a}^{ \pm}\right)^{-2} \int_{-\infty}^{+\infty}\left\langle\tilde{\Phi}^{ \pm f}, S_{x}\right\rangle d x, \\
\tilde{\tau}^{ \pm}=-\left(\tilde{a}^{ \pm}\right)^{-2} \int_{-\infty}^{+\infty}\left\langle\tilde{\Psi}^{ \pm f}, S_{x}\right\rangle d x, \\
\delta \tilde{Q}^{ \pm}= \pm 2 i\left(\tilde{a}^{ \pm}\right)^{-2} \int_{-\infty}^{+\infty}\left\langle\tilde{\Phi}^{ \pm f}, \tilde{\Lambda}_{-}^{*} \delta S\right\rangle d x, \\
\delta \tilde{\tau}^{ \pm}= \pm 2 i\left(\tilde{a}^{ \pm}\right)^{-2} \int_{-\infty}^{+\infty}\left\langle\tilde{\Psi}^{ \pm f}, \tilde{\Lambda}_{+}^{*} \delta S\right\rangle d x .
\end{gathered}
$$

Proof. It is well known that the Killing form is invariant under the adjoint group action $\operatorname{Ad}(h)$, i.e., $\langle\operatorname{Ad}(h) X, \operatorname{Ad}(h) Y\rangle=\langle X, Y\rangle$. We have found how the quantities $\widetilde{\delta q}=\operatorname{Ad}\left(\psi_{0}^{-1}\right) \delta q, \tilde{q}=\operatorname{Ad}\left(\psi_{0}^{-1}\right) q$ are expressed through $S$ and $\delta S$. That is why the statement of the theorem follows from the formulae (2.4).

Theorem 2. There exist the following expansions over the sets $\left\{\tilde{\Psi}^{ \pm f}\right\},\left\{\tilde{\Phi}^{ \pm f}\right\}$, and $\{\tilde{P}, \tilde{Q}\}$ :

$$
\begin{aligned}
\frac{1}{4}\left[S, S_{x}\right] & =\frac{1}{\pi} \int_{-\infty}^{+\infty} d \lambda\left(\tilde{\varrho}^{+} \tilde{\Psi}^{+f}-\tilde{\varrho}^{-} \tilde{\Psi}^{-f}\right) \\
& =\frac{1}{\pi} \int_{-\infty}^{+\infty} d \lambda\left(\tilde{\tau}^{+} \Phi^{+f}-\tilde{\tau}^{-} \tilde{\Phi}^{-f}\right) \\
& =\int_{-\infty}^{+\infty} d \lambda \tilde{P}(x, \lambda), \\
\Lambda_{+}[S, \delta S] & =-\frac{2 i}{\pi} \int_{-\infty}^{+\infty} d \lambda\left(\delta \tilde{\varrho}^{+} \tilde{\Psi}^{+f}+\delta \tilde{\varrho}^{-} \tilde{\Psi}^{-f}\right), \\
\Lambda_{-}[S, \delta S] & =\frac{2 i}{\pi} \int_{-\infty}^{+\infty} d \lambda\left(\delta \tilde{\tau}^{+} \tilde{\Phi}^{+f}+\delta \tilde{\tau}^{-} \tilde{\Phi}^{-f}\right), \\
\Lambda[S, \delta S] & =-2 \int_{-\infty}^{+\infty} d \lambda(\delta \tilde{\hat{q}} \tilde{P}+\delta \tilde{\hat{p}} \tilde{Q}),
\end{aligned}
$$

where $\tilde{\hat{q}}=\frac{i}{2} \log \tilde{b}^{+}\left(\tilde{b}^{-}\right)^{-1}, \tilde{\hat{p}}=-\frac{1}{\pi} \log \left(1+\tilde{\varrho}^{+} \tilde{\varrho}^{-}\right)$.

Proof. Let us apply $\operatorname{Ad}\left(\psi_{0}^{-1}\right)$ to the expansions (2.9) and (2.10). As in the proof of the previous theorem we express $\tilde{q}$ and $\tilde{\delta q}$ through $S$ and $\delta S$ and arrive to $(4.2 \mathrm{a}-\mathrm{c})$ and (4.3a). As to the expansions (4.3b-c) Lemma 4 shows that an additional term proportional to $\delta \log a^{+}(0)$ will appear in the left-hand side. However, one can see that analogical terms exist in the right-hand side as well, due to the fact that

$$
\delta \tilde{\tau}^{ \pm} \tilde{\Phi}^{ \pm f}=\left[\delta \tau^{ \pm}+2 \tau^{ \pm} \delta \log a^{+}(0)\right] \operatorname{Ad}\left(\psi_{0}^{-1}\right) \Phi^{ \pm f}
$$

and $\delta \tilde{\hat{q}}=\delta \hat{q}-i \delta \log a^{+}(0)$. It is easy to see that the cancellation of these terms follows from $(4.2 \mathrm{~b}-\mathrm{c})$. 
Now we have overcome all the difficulties and are able to reformulate the results I-IV in the case of the HF-type equations. We would like to note that as all of them are consequences of the expansions (2.9) and (2.10), the corresponding results for the HF-type equations may be proved just in the same way starting from the expansions (4.2) and (4.3).

I. The inversion formulae (4.1) together with the expansions (4.2) and (4.3) show that the quantity $M(x)=\frac{1}{4}\left[S, S_{x}\right]$ can be recovered uniquely from the set of the scattering data $\widetilde{R}_{\varrho}$ and vice versa. In its turn the potential $S(x)$ is determined uniquely from $M(x)$ as a solution of the linear problem

$$
S_{x}=[S, M]
$$

with the boundary condition $\lim _{x \rightarrow+\infty} S(x)=\sigma_{3}$. The fact that in the same time $\lim _{x \rightarrow-\infty} S(x)=\sigma_{3}$ follows from the restrictions $\varrho^{ \pm}(0)=0$. Thus the interpretation of the ISM as a generalized Fourier transform has a general character and does not depend on the choice of the gauge for the linear problem $L(q, \lambda) \psi=0$.

II. It is not difficult to find that the class of the solvable NLEEs, gauge equivalent to Eqs. (2.15) is given by:

$$
2 F\left(\tilde{\Lambda}_{ \pm}\right) \tilde{\Lambda}_{ \pm}\left[S, S_{t}\right]+i G\left(\tilde{\Lambda}_{ \pm}\right)\left[S, S_{x}\right]=0 .
$$

Needless to say that these equations are equivalent to the linear equations (2.16) for the scattering data, and that as before one can write in (4.5) the operator $\tilde{\Lambda}$ instead of $\tilde{\Lambda}_{ \pm}$without changing anything.

Sometimes one prefers to describe the same set of equations by means of the adjoint operators $\tilde{\Lambda}_{+}^{*}$. If we introduce $F_{1}(\lambda)=2 \lambda F(\lambda)$ and $G_{1}(\lambda)=i G(\lambda)$, then with the help of Lemma 3 we have

$$
F_{1}\left(\tilde{\Lambda}_{+}^{*}\right) S_{t}+G_{1}\left(\tilde{\Lambda}_{+}^{*}\right) S_{x}=0 .
$$

Besides, written like that the equations are invariant under the permutation $x \leftrightarrow t$, $F_{1} \leftrightarrow G_{1}$.

Remark 6. A similar arguments applied to Eqs. (2.15) show that they may be written in the symmetric form as well:

$$
F_{2}\left(\Lambda_{+}^{*}\right) q_{t}+G_{2}\left(\Lambda_{+}^{*}\right) q_{x}=0,
$$

where $F_{2}=\frac{1}{2} F$ and $G_{2}(\lambda)=\frac{i}{4} \lambda^{-1} G(\lambda)$.

Below we give some examples of the HF-type equations, making the same choices for $F$ and $G$ as in Sect. 2, and thus obtaining the corresponding equivalent pairs of equations. First of all, for $F(\lambda)=1, G(\lambda)=4 i \lambda^{2}$, one gets the HFE (1.4) which is gauge-equivalent to the NLSE (1.2). The choice (2.1) for $F$ and $G$ leads to the equation

$$
S_{t}+S_{x x x}-\left\langle S, S_{x x x}\right\rangle S+\frac{3}{2}\left\langle S_{x}, S_{x}\right\rangle S_{x}=0,
$$

equivalent to the $\mathrm{mKdV}$ equation (2.18).

The counterparts of (2.21) and (2.22) are a bit more difficult to find, but since one is able to calculate explicitly the matrix $\psi_{0}$ through $v(x, t)$ we find that both sine- and sinh-Gordon equations are equivalent to

$$
S_{x t}+\left\langle S_{t}, S_{x}\right\rangle S+\frac{1}{2}\left[\sigma_{3}, S\right] \sigma_{3}=0,
$$


with different additional conditions however:

$$
\left\langle S, \sigma_{1}\right\rangle=0, \quad\left\langle S, \sigma_{2}\right\rangle=0
$$

for the $V_{+}$and $V_{-}$cases, respectively $\left(\sigma_{1,2}\right.$ are the Pauli matrices).

The following remark concernes the Lax representations of the sine-Gordon and sinh-Gordon equations. These equations may be written in the form (1.3), where $q$ is subject of the restrictions (2.20) and $M=i \frac{\partial}{\partial t}+\frac{1}{\lambda} S(x, t)$. The compatibility condition (1.3) leads to an interrelation between $q$ and $S$ which may be solved explicitly. Thus choosing $q$ as an independent variable and solving for $S$ we obtain (2.21) or (2.22) depending on the sign of $\varepsilon$. But we can choose $S$ as the independent variable and express $q$ through it. This directly leads to (4.9). Therefore, we see that in this example the gauge transformation interchanges the operators $M$ and $L$ in the Lax representation.

III. The compact formulae (2.25) is a starting point for obtaining the series of conservation laws with a local densities for the HF-type equations. From (3.1) and (3.2) it follows that $\log \tilde{a}^{+}=\log a^{+}-\log a^{+}(0)$. Therefore, the generating functionals for the NLS-type equations and HF-type equations differ only by the quantity $\log a^{+}(0)$, which is a conservation law by itself. Applying to the integrand of (2.25) the transformation $\operatorname{Ad}\left(\psi_{0}^{-1}\right)$ we obtain:

$$
c_{m}=-\frac{1}{4 m} \int_{-\infty}^{+\infty} d x \int_{x}^{+\infty}\left\langle S_{y}, \tilde{\Lambda}_{+}^{m}\left[S, S_{y}\right]\right\rangle d y \equiv \int_{-\infty}^{+\infty} \varrho_{m}(x) d x .
$$

Utilizing the explicit form of $\tilde{\Lambda}_{+}$, one is able to obtain (up to numerical factors and $x$-derivative terms) the first three conserved densities:

$$
\begin{gathered}
\varrho_{1}=\left\langle S_{x}, S_{x}\right\rangle, \quad \varrho_{2}=\left\langle S,\left[S_{x}, S_{x x}\right]\right\rangle, \\
\varrho_{3}=5\left\langle S_{x}, S_{x}\right\rangle^{2}-4\left\langle S_{x x}, S_{x x}\right\rangle .
\end{gathered}
$$

At first sight $\varrho_{3}$ differs from the expression obtained in [12] which contains rational dependence on $S_{x}$, i.e., a factor $\left\langle S_{x}, S_{x}\right\rangle^{-1}$. But, if $S_{x} \neq 0$, then $S, S_{x}$, and $\left[S, S_{x}\right]$ are linearly independent and form a basis in $\operatorname{sl}(2, \mathbb{C})$. Expanding $S_{x x}$ over this basis one can check that

$$
\varrho_{3}=\left\langle S_{x}, S_{x}\right\rangle^{2}-\left(\frac{d}{d x}\left\langle S_{x}, S_{x}\right\rangle^{2}-\left\langle S_{x x},\left[S, S_{x}\right]\right\rangle\right)\left\langle S_{x}, S_{x}\right\rangle^{-1},
$$

which coincides with the quantity in [12]. Besides, since the operators $\tilde{\Lambda}_{ \pm}$depend polynomially on $S$, it is obvious that all the densities $\varrho_{m}(x)$ will depend polynomially in $S$ and its $x$-derivatives. The fact that $\varrho_{m}$ are also local functions in $S$ deserves special attention and will be proved elsewhere.

IV. Let us describe now the interrelation between the hierarchies of symplectic structures. The mapping $q: \tilde{\mathscr{M}} \rightarrow \mathscr{M}^{0}$ is nonsingular and therefore we can define the following hierarchy of symplectic forms on $\tilde{\mathscr{M}}$ (it is of course the same hierarchy in "different coordinates")

$$
q^{*} \omega_{F}(\tilde{X}, \tilde{Y})=\omega_{F}\left(q^{\prime}(S) \tilde{X}, q^{\prime}(S) \tilde{Y}\right) .
$$


A simple calculation shows that

$$
\begin{aligned}
q^{*} \omega_{F}(\tilde{X}, \tilde{Y})= & i \int_{-\infty}^{+\infty} d x\left\langle\tilde{X}, F(\tilde{\Lambda}) \tilde{\Lambda}^{2}[S, \tilde{Y}]\right\rangle \\
& +2 i\left(d \log a^{+}(0)\right) \wedge \beta_{F}(\tilde{X}, \tilde{Y}) ; \quad \tilde{X}, \tilde{Y} \in \mathscr{S}_{S},
\end{aligned}
$$

where the 1 -form $\beta_{F}$ is given by:

$$
\beta_{F}(\tilde{X})=\frac{i}{4} \int_{-\infty}^{+\infty}\left\langle\tilde{X}, F(\tilde{\Lambda}) \tilde{\Lambda}\left[S, S_{x}\right]\right\rangle d x .
$$

It is not difficult to understand that the hierarchy of symplectic structures corresponding to the HF-type equations is determined by the following 2-forms:

$$
\tilde{\omega}_{G}(\tilde{X}, \tilde{Y})=i \int_{-\infty}^{+\infty}\langle\tilde{X}, G(\tilde{\Lambda})[S, \tilde{Y}]\rangle d x
$$

and therefore the formula (4.15) gives the connection between the two hierarchies.

The case $F=F_{0}=\lambda^{-2}$ is of particular interest since then the first term in the right-hand side of (4.15) is exactly the canonical symplectic form $\tilde{\omega}_{0}$. We have then:

$$
q^{*} \omega_{F_{0}}=\tilde{\omega}_{0}+2 i\left(d \log a^{+}(0)\right) \wedge \beta_{F_{0}} .
$$

Making use of the dispersion relations and the expansion formula (4.2c) one can write down this equation in an equivalent form:

$$
\begin{gathered}
q^{*} \omega_{F_{0}}=\tilde{\omega}_{0}-\frac{1}{2} d H_{-1} \wedge d H_{-2} \\
H_{-1}=\text { p.v. } \int_{-\infty}^{+\infty} \frac{\tilde{\hat{p}}(\lambda)}{\lambda} d \lambda, \quad H_{-2}=\text { p.v. } \int_{-\infty}^{+\infty} \frac{\tilde{\hat{p}}(\lambda)}{\lambda^{2}} d \lambda .
\end{gathered}
$$

For the first time this result has been obtained in [10] comparing the expressions for $\tilde{\omega}_{0}$ and $\omega_{0}$ in terms of the scattering data.

\section{The Geometrical Interpretation of the $\Lambda$-Operator Theory and the Gauge Transformations}

It is known that the $\Lambda$-operator theory has a beautiful geometrical interpretation, based on the fact that $\Lambda^{*}$ can be regarded as a tensor field on an infinitedimensional symplectic manifold, possessing some special geometrical properties. We shall outline the main points, for more details, see [11, 20, 21$]$.

First of all one can consider $\mathscr{M}$ (the phase space for the NLS-type equations) as an infinite-dimensional manifold, $q$ being the coordinate function on it. As in fact $\mathscr{M}=\mathscr{S}_{\sigma}$ is a linear space, it is evident that the tangent space $T_{q}$ at the point $q \in \mathscr{M}$ is equal to $\mathscr{S}_{\sigma}$ and the tangent bundle $T(\mathscr{M})=\mathscr{M} \times \mathscr{S}_{\sigma}$. An arbitrary mapping $X: \mathscr{M} \rightarrow \mathscr{S}_{\sigma}$ can be considered as a vector field on $\mathscr{M}$. In the same way the mapping of the type $A: \mathscr{M} \rightarrow \mathscr{L}\left(\mathscr{S}_{\sigma}, \mathscr{S}_{\sigma}\right)$ can be regarded as an $(I, I)$ tensor field on $\mathscr{M}$. [Here as usual $\mathscr{L}\left(\mathscr{S}_{\sigma}, \mathscr{S}_{\sigma}\right)$ denotes the space of all linear operators on $\left.\mathscr{S}_{\sigma} \cdot\right]$ For example, with the help of the formulae (3.17) one is able to construct for any $q \in \mathscr{M}$ the 
following operator:

$$
\begin{aligned}
\Lambda_{q}^{*}= & \frac{1}{2}\left(\Lambda_{+}^{*}+\Lambda_{-}^{*}\right)=\frac{i}{4}\left[\sigma_{3}, \frac{d}{d x}\right] \\
& +\frac{i}{2}\left[\sigma_{3}, q\right]\left(\int_{+\infty}^{x}+\int_{-\infty}^{x}\right)\langle q, \cdot\rangle d y,
\end{aligned}
$$

or in other words $\Lambda^{*}$ is a tensor field on $\mathscr{M}$. For every $q$ as a cotangent space $T_{q}^{*}$ we shall take $\mathscr{S}_{\sigma}$ and put it into duality with $T_{q}$ by means of the bilinear form $g$, see (3.13).

We have already mentioned that the 2 -form $\omega_{0}$, see (3.14) defines the canonical symplectic structure on $\mathscr{M}$. For our purpose, however, it is more convenient to describe the symplectic structure with the help of the so-called symplectic "operator" $H$. For an arbitrary point $q \in \mathscr{M}, H$ can be regarded as a linear mapping from the cotangent space $T_{q}^{*}$ to the tangent space $T_{q}$. The tensor field $H$ is completely determined through the requirement

$$
\alpha(H \beta)=\omega_{0}(H \alpha, H \beta) ; \quad \alpha, \beta \in T_{q}^{*}=\mathscr{S}_{\sigma} .
$$

We prefer the tensor field $H$ rather than the 2-form $\tilde{\omega}_{0}$ because the expression of $H$ is extremely simple:

$$
H \alpha=\frac{i}{4}\left[\sigma_{3}, \alpha\right], \quad \alpha \in \mathscr{S}_{\sigma}
$$

Below we shall need also the notion of the Lie bracket of two vector fields $X$ and $Y$. We shall denote it through $[X, Y]^{L}$. It is defined by the relation:

$$
[X, Y]^{L}=Y^{\prime}(q) X-X^{\prime}(q) Y .
$$

After these preliminaries let us pass to the properties of the tensor field $\Lambda^{*}$, given by the expression (5.1). It was noticed that $\Lambda^{*}$ satisfies a number of conditions, see [11]. The first one is the so-called Nijenhuis condition, [22]. It means that whatever the two vector fields $X$ and $Y$ are, the following equation holds:

$$
\left(\Lambda^{*}\right)^{2}[X, Y]^{L}+\left[\Lambda^{*} X, \Lambda^{*} Y\right]^{L}=\Lambda^{*}\left(\left[\Lambda^{*} X, Y\right]^{L}+\left[X, \Lambda^{*} Y\right]^{L}\right)
$$

The other conditions are the so-called coupling conditions and they describe the interrelation between the symplectic structure and $\Lambda^{*}$. The first of them reads

$$
H \Lambda=\Lambda^{*} H,
$$

and evidently is the already established property (3.23). The second coupling condition is more complicated. It can be written in the following form:

$$
\begin{aligned}
g\left(X, \Lambda^{* \prime}(Z) H Y\right) & -g\left(X, \Lambda^{* \prime}(H Y) Z\right)+g\left(Y, \Lambda^{*^{\prime}}(H X) Z\right) \\
& +g\left(Y, \Lambda^{*} H^{\prime}(Z) X\right)-g\left(Y, H^{\prime}\left(\Lambda^{*} Z\right) X\right)=0
\end{aligned}
$$


for every three vector fields $X, Y$, and $Z$. All these properties are sometimes referred to as the symplectic Kähler structure, [11]. It may be shown, that it follows from them that the equations

$$
q_{t}=H \Lambda^{n} q, \quad n=1,2, \ldots,
$$

are Hamiltonian ones and the right-hand sides define commuting flows [compare with (2.15)]. Some other important facts from the theory of the integrable equations also find their natural geometrical explanation within this scheme.

Our intention is to show that the geometrical properties of the operator $\Lambda^{*}$ do not depend upon the gauge. The gauge transformation maps the phase space $\mathscr{M}^{0} \subset \mathscr{M}$ into the space

$$
\tilde{\mathscr{M}}=\left\{S(x), S^{2}=\mathbb{1}, \lim _{x \rightarrow \pm \infty} S(x)=\sigma_{3}\right\} .
$$

First of all, let us note that the submanifold $\mathscr{M}^{0}$ is an invariant submanifold for the operator field $\Lambda^{*}$. Indeed, $\mathscr{M}^{0}$ is defined as the set of points $q \in \mathscr{M}$, satisfying the constraints $\tau^{ \pm}(0)=0\left(\varrho^{ \pm}(0)=0\right)$. Therefore, $X \in T_{q}$ is a tangent vector for the submanifold $\mathscr{M}^{0}$ if $d \tau^{ \pm}(0)(X)=0$, (or $\left.d \varrho^{ \pm}(0)(X)=0\right)$. From the inversion formulae (2.4) it follows that $d \tau^{ \pm}(0)\left(\Lambda_{+}^{*} X\right)=d \varrho^{ \pm}(0)\left(\Lambda_{-}^{*} X\right)=0$ for an arbitrary $X \in \mathscr{S}_{\sigma} . \mathscr{M}^{0}$ is then an invariant submanifold for $\Lambda_{+}^{*}$ and $\Lambda_{-}^{*}$, and hence for $\Lambda^{*}$.

Now, the manifold $\tilde{\mathscr{M}}$ is also an infinite dimensional manifold, and $T(\tilde{\mathscr{M}})$ $=\bigcup_{S \in \tilde{\mathscr{M}}} \mathscr{S}_{S}$, i.e., the tangent space at the point $S \in \tilde{\mathscr{M}}$ is exactly $\mathscr{S}_{S}$. As before $T_{S}^{*}=\mathscr{S}_{S}$ and the duality is given by the bilinear form $\tilde{g}$, see (3.15). The canonical symplectic structure on $\tilde{\mathscr{M}}$ is defined by the 2 -form $\tilde{\omega}_{0},(3.16)$. Just as before one easily finds the symplectic operator $\tilde{H}$ :

$$
\tilde{H} \tilde{\alpha}=\frac{i}{4}[S, \tilde{\alpha}], \quad \tilde{\alpha} \in T_{S}^{*}=\mathscr{S}_{S} .
$$

Next we consider the operator field $\tilde{\Lambda}^{*}$ defined as:

$$
\tilde{\Lambda}_{S}^{*}=\frac{1}{2}\left(\tilde{\Lambda}_{+}^{*}+\tilde{\Lambda}_{-}^{*}\right)=\frac{i}{4}\left[S, \frac{d}{d x} \cdot\right]-\frac{i}{8} S_{x}\left(\int_{+\infty}^{x}+\int_{-\infty}^{x}\right)\left\langle\left[S, S_{y}\right], \cdot\right\rangle d y
$$

and are able to formulate the following

Theorem 3. The tensor field $\tilde{\Lambda}^{*}$ satisfies the Nijenhuis condition and is coupled with the symplectic structure on $\tilde{\mathscr{M}}$.

The proof of the first of the coupling conditions coincides with the proof of the relation (3.24). As to the Nijenhuis condition and the second coupling condition, we regret to say that we have no better proof but a direct verification. This is one more reason that the explicit form of $\tilde{\Lambda}^{*}$ is indispensible. The verification, although laborious, is quite straightforward and we shall omit it.

Thus the geometrical interpretation has also a general character, and of course so are all the results that are consequences of the symplectic Kähler structure. 


\section{Conclusions}

In the present paper we have considered the simplest non-trivial example of gaugeequivalent linear problems. The method, however, can be extended in order to investigate more complicated examples. Some preliminary results of this type have been reported in a conference talk [23].

Acknowledgements. The authors are grateful to Prof. V. G. Makhankov for his interest and support and to Dr. P.P.Kulish for the useful discussions. Also we would like to thank the referee whose remarks helped us to improve the manuscript.

\section{References}

1. Zakharov, V.E., Manakov, S.V., Novikov, S.P., Pitaevskii, I.I.: Soliton theory: the inverse problem method. Moscow: Nauka 1981

2. Ablowitz, M., Kaup, D.J., Newell, A.C., Seegur, H.: The inverse scattering problem-Fourier analyses for non-linear problems. Stud. Appl. Math. 53, 249-315 (1974)

3. Kaup, D.J.: Closure of the Zakharov-Shabat eigenstates. J. Math. Anal. Appl. 54, 849-864 (1976)

4. Gerdjikov, V.S., Khristov, E.Kh.: On the expansions over the products of solutions of two Dirac systems. Mat. Zametki 28, 501-512 (1980)

5. Kaup, D.J., Newell, A.C.: Soliton equations, singular dispersion relations and moving eigenvalues. Adv. Math. 31, 67-100 (1979)

6. Gerdjikov, V.S.., Khristov, E.Kh.: On the evolution equations, solvable by the inverse problem method. I. Spectral theory. Bulg. J. Phys. 7, 28-41 (1980); II. Hamiltonian structures and Bäcklund transformations. Bulg. J. Phys. 7, 119-133 (1980)

7. Calogero, F., Degasperis, A.:Nonlinear evolution equations solvable by the inverse spectral transform. I. Nuovo Cimento 32 B, 201-242 (1976)

8. Flaschka, H., Newell, A.C.: Integrable systems of non-linear evolution equations. Lecture Notes in Physics, Vol. 38, pp. 355-440. Berlin, Heidelberg, New York: Springer 1975

9. Faddeev, L.D.: In: Solitons. Bullough, R.K., Caudrey, P. (eds.). A Hamilton interpretation of the inverse scattering method. Top. Current Phys. 17, 339-354 (1980)

10. Kulish, P.P., Reyman, A.G.: A hierarchy of symplectic forms for Schrödinger and Dirac equations on a line. Zap. Nauchn. Semin. LOMI 77, 134-147 (1978)

11. Magri, F.: A geometrical approach to the non-linear solvable equations. In: Lecture Notes in Physics, Vol. 120, pp. 239-265. Berlin, Heidelberg, New York: Springer 1980

12. Zakharov, V.E., Takhtadjian, L.E.: The equivalence of the nonlinear Schrödinger and the Heisenberg ferromagnet equations. Theor. Math. Phys. 38, 26-35 (1979)

13. Zakharov, V.E., Mikhailov, A.V.: Relativistically invariant two-dimensional field theory models, integrable by the inverse problem method. JETPh. 74, 1953-1973 (1978)

14. Makhankov, V.G., Pashaev, O.K.: On the gauge equivalence of the Landau-Lifshitz and nonlinear Schrödinger equations on symmetric spaces. Phys. Lett. A, 95A, 95-100 (1983)

15. Fordy, A., Kulish, P.P.: Non-linear Schrödinger equations and simple Lie algebras. Commun. Math. Phys. 89, 427-443 (1983)

16. Gerdjikov, V.S., Kulish, P.P.: The generating operator for the $n \times n$ linear system. Physica D, D 3, 549-564 (1981)

17. Gerdjikov, V.S., Yanovski, A.B.: Gauge covariant formulation of the generating operator. I. The Zakharov-Shabat system. Phys. Lett. 103A, 232-236 (1984) 
18. Konopel'chenko, B.C., Dubrovsky, V.G.: General $N^{\text {th }}$ order differential spectral problem: General structure of the integrable equations, nonuniqueness of recursion operator and gauge invariance. INPh preprint No.83-26, Novosibirsk (1983)

19. Bruschi, M., Levi, D., Ragnisco, O.: The chiral field hierarchy. Preprint of the Institute of Physics "G. Marconi," Rome, No.271 (1981)

20. Florko, B., Yanovski, A.B.: On Magri's theorem for complete integrability. JINR preprint E583-813, Dubna (1983)

21. Gel'fand, I.M., Dorfman, I.I.: The Schouten bracket and the Hamiltonian operators. Funkts. Anal. Prilozh. 14, 3, 71-74 (1980)

22. Frölicher, A., Nijenhuis, A.: Theory of vector-valued differential forms. Nederl. Acad. Westench. Proc. 59A, 338-359 (1965)

23. Gerdjikov, V.S., Yanovski, A.B.: Gauge covariant formulation of the generating operator for the non-linear evolution equations on symmetric spaces. Report on the III International Symposium on Selected Problems of Statistical Mechanics, Dubna, August 1984, JINR preprint D17-84-407, p. 43, Dubna (1984)

24. Eichenherr, H., Forger, M.: On the symmetry of the non-linear sigma models. Nucl. Phys. B 155, 381-393 (1979)

Communicated by Ya. G. Sinai

Received January 15, 1985; in revised form November 4, 1985 